\title{
Lumen
}

Selected Proceedings from the Canadian Society for Eighteenth-Century Studies

\section{Femmes en public et femmes publiques dans le Tableau de Paris de Mercier}

\section{Laurence Mall}

Volume 24, 2005

Indigenes and Exoticism

Indigènes et exotisme

URI : https://id.erudit.org/iderudit/1012177ar

DOI : https://doi.org/10.7202/1012177ar

Aller au sommaire du numéro

Éditeur(s)

Canadian Society for Eighteenth-Century Studies / Société canadienne d'étude du dix-huitième siècle

ISSN

1209-3696 (imprimé)

1927-8284 (numérique)

Découvrir la revue

Citer cet article

Mall, L. (2005). Femmes en public et femmes publiques dans le Tableau de Paris de Mercier. Lumen, 24, 93-104. https://doi.org/10.7202/1012177ar

Copyright (c) Canadian Society for Eighteenth-Century Studies / Sociéte canadienne d'étude du dix-huitième siècle, 2005
Ce document est protégé par la loi sur le droit d'auteur. L'utilisation des services d'Érudit (y compris la reproduction) est assujettie à sa politique d'utilisation que vous pouvez consulter en ligne.

https://apropos.erudit.org/fr/usagers/politique-dutilisation/ 


\section{Femmes en public et femmes publiques dans le Tableau de Paris de Mercier}

A la fin du livre IV du Tableau de Paris, dans le chapitre consacré au compte rendu de son livre donné dans le Courrier de l'Europe (du 3 juillet 1781) Mercier cite cette remarque: «Dans vingt chapitres, il parle des femmes comme si Paris n'était qu'un lieu de prostitution, où la pudeur et la décence n'osent plus se montrer» (CCCLVII, I, 991) ${ }^{1}$. Et certes dans le texte du grand piéton de Paris les péripatéticiennes figurent en bonne place. Mais c'est aux femmes de toutes conditions que Mercier fait une large place dans son immense ouvrage publié entre 1781 et 1788 (l'ouvrage en douze tomes comporte plus de 3000 pages dans l'édition moderne). L'intéressent avant tout «la physionomie morale» de la capitale et ses nuances fugitives (Préface, I, 14). La modernité est embrassée en ce qu'il nomme des «figures vivantes»: «je me suis occupé de la génération actuelle» (Préface, I, 17). La sensibilité de Mercier aux microcontextes, sa reconnaissance des déterminants à la fois collectifs et spécifiques pesant sur l'individu et l'intelligence des variantes qu'il manifeste contribuent à l'admiration que lui témoignent de grands spécialistes contemporains de l'histoire sociale du XVIII ${ }^{e}$ siècle, tels Arlette Farge ou Daniel Roche. Ce sont aussi ces qualités qui confèrent à ses considérations sur les femmes leur exceptionnel intérêt. Le Tableau offre des femmes en général un traitement remarquable par rapport au théâtre ou au roman ou encore au genre épistolaire où l'accent est mis sur la vie privée et l'intériorité des héroïnes, sur leur individualité portée par une parole singulière. Ce traitement diffère aussi fortement de l'univers normatif et abstrait des traités de morale ou des vastes perspec-

1 Toutes les citations du Tableau de Paris de Louis-Sébastien Mercier sont tirées de l'édition en deux volumes établie sous la direction de Jean-Claude Bonnet, Paris, Mercure de France, 1994. Suivant la citation, sont indiqués entre parenthèses, dans cet ordre, le numéro du chapitre, celui du volume et enfin la page. 
tives des essais psycho-anthropologiques comme celui de Diderot («Des femmes») ou ceux de Laclos. Mercier a bien davantage le goût du concret, du contingent et de l'accidentel; il s'intéresse non pas tant au particulier qu'au spécifique, et ses conclusions sont provisionnelles et irrégulières, loin des thèses carrées. Ne négligeons pas enfin cette remarque de la préface: «ll eût été facile de faire de ce Tableau un livre satirique; je m'en suis sévèrement abstenu» $(I, 17)$, même si cette intention se perd parfois dans les irrésistibles élans d'une plume moqueuse ${ }^{2}$. Le «je» de Mercier est très présent au fil des pages, et son regard sur les femmes, s'il est porté sur des situations objectives, est personnel, souvent lourd d'humeurs, et évidemment informé par sa propre sensibilité masculine (ses «j'aime/je n'aime pas» sont déguisés de façon transparente sous des «on souffre à voir» ou «on aime à voir» telle sorte de femmes, telle parure, tel comportement, etc). Mais l'intérêt de ses considérations sur les femmes dépasse très largement la dimension personnelle (par ailleurs attachante). Je chercherai ici à en cerner une partie en mettant en valeur chez Mercier sa double perspective de moraliste et de «sociologue avant la lettre ${ }^{3} »$.

Mercier est indéniablement influencé par la vision rousseauiste de la vie urbaine et des femmes. La longue tradition qui fait de Paris une Babylone moderne a été relancée avec grande énergie et grand succès par la série des lettres sur Paris dans La Nouvelle Héloïse de 1761. Mercier forme à son tour un important maillon de la chaîne qui lie Balzac au $\mathrm{XVIII}^{\mathrm{e}}$ siècle et par laquelle Paris est constitué en «mythe moderne ${ }^{4}$ ». Pour lui comme pour tant d'autres, c'est dans ce lieu quintessentiel de corruption et de perdition que pullulent les abus les plus graves et que les vices triomphent; $c^{\prime}$ est dans ce "gouffre où se fond l'espèce humaine" (III, I, 32) que s'exhibent le plus crûment les différences de condition mais aussi que s'opèrent de dramatiques métamorphoses et que menacent de

2 Voir par exemple le chapitre «Plumasserie», où les «têtes emplumées offrent de loin une ondulation brillante; mais une pluie survient, et ces plumes colorées qui jouaient avec les zéphirs tombent, s'affaissent et impriment à la figure de toutes ces femmes la triste image de poules mouillées», etc. (DCCXXXI, II, 670-71).

3 Daniel Roche salue chez Mercier la pratique d'une «sociologie avant la lettre," $L a$ France des Lumières, Paris, Fayard, 1993, p. 595.

4 Roger Caillois, «Paris, mythe moderne», dans Le Mythe et l'homme, Paris, Gallimard (1938), coll. «Idées», 1972, 150-171. Voir aussi Henry F. Majewski, The Preromantic Imagination of L.-S. Mercier, New York, Humanities Press, 1971, p. 115-136. 
toxiques mélanges sociaux. C'est à Paris que s'expose le plus dangereusement à la tentation la vertu des hommes, et surtout celle des femmes en son sens plus restreint. C'est là qu'elles sont à la fois le plus sordidement asservies, ou le plus insolemment libres selon leur condition et le hasard. Tout cela, le chroniqueur des rues affirme en voir ou en lire les signes tout autour de lui, au grand jour. N'est-ce pas aisé, puisque les Parisiennes semblent passer leur temps à l'extérieur? «Elles ont grand besoin de voir et d'êtres vues» (CDXVIII, I, 1156). Or cette jouissance de l'ostentation est un mal. Rousseau avait fameusement proclamé que «toute femme qui se montre est une femme qui se déshonore ${ }^{5}$ » et $\mathrm{qu}^{\prime}$ «une femme hors de sa maison perd son plus grand lustre, et dépouillée de ses vrais ornements, elle se montre avec indécence ${ }^{6}$ ».

Cette position absurdement rigoriste et irrémédiablement archaïque lorsqu'elle est détachée de l'enchaînement précis de son contexte dans la Lettre à d'Alembert, Mercier certes ne l'adopte pas de front. Mais il procède de façon appuyée à une semblable valorisation de la vertueuse épouse bourgeoise, chaste ménagère: «les vertus domestiques, les plus pénibles de toutes, mais aussi les plus satisfaisantes, voilà leur véritable gloire» (DCXCIV, II, 565). Que l'on songe au chapitre, étonnant dans son absence d'ironie, intitulé «Nos grand-mères», où se trouvent concentrés nombre de clichés sur le bon vieux temps où les femmes, "contentes d'une royauté domestique», "n'étaient pas aussi répandues; on ne les voyait pas incessamment hors de leurs maisons. [...] Elles entretenaient le bon ordre et l'harmonie dans leur empire, fixaient le bonheur dans leurs foyers [...]» (LIV , I, 145) ${ }^{7}$. Aujourd'hui même, il existe heureusement «une classe de femmes très respectables; c'est celle du second ordre de la bourgeoisie. Attachées à leurs maris et à leurs enfants, soigneuses, économes, attentives à leurs maisons, elles offrent le modèle de la sagesse et du travail. [...] On ne les aperçoit pas, et cependant elles sont à Paris l'honneur de leur sexe» (CCXLIX, I, 627). A la veille de la Révolution Mercier se situe de toute évidence dans le courant idéologique tant affermi par Rousseau et qui dominera non seulement les débats sur les

5 Jean-Jacques Rousseau, Lettre à d'Alembert sur les spectacles, dans Oeuvres complètes, éd. B. Gagnebin et M. Raymond, Paris, Gallimard, «Bibliothèque de la Pléiade», 1995, vol. V, p. 76.

6 Lettre à d'Alembert, p. 80.

7 Voir aussi dans le chapitre intitulé «De L'idole de Paris, le joli» un modèle de vie primitive, offert favorablement, où «les femmes sont séquestrées de la société [...]. Elles se bornent à donner des citoyens, à les élever, à gouverner un ménage» (CCLIV, I, 637). 
femmes mais leur condition effective lorsqu'elles seront plus qu'encouragées à abandonner la sphère publique pour demeurer au foyer. Sans doute verra-t-on une différence entre se montrer aux promenades et avoir sa place sur l'agora, entre simple présence en public et vie publique dans un sens politique. Mais Michèle Perrot précise justement que la Cité «oscille entre deux pôles: l'un, juridique et politique, concerne les droits et les devoirs qui confèrent un statut, celui du citoyen, au sens de la Polis grecque. L'autre, plus spatial, désigne la Ville, et plus encore son coeur, son centre. Entre les deux sens, le politique et le spatial, les recoupements et les recouvrements sont incessants ${ }^{8} \gg$. La réprobation envers la simple présence des femmes dans la rue ne peut bien augurer de leur participation à la res publica.

Mercier n'est pas comme Rousseau un grand penseur politique et on ne saurait lui attribuer de thèses générales; lui-même affirme: «je n'ai presque rien donné à la réflexion du philosophe» (Préface, I, 17). Pour Rousseau par contre, la critique de la participation des femmes à la vie publique est rigoureusement déterminée par l'ensemble de son système politique et, en l'occurrence, par sa conception du citoyen: ce dernier ne peut bien remplir ses devoirs civiques et politiques que si sa vie privée est saine et stable, ce qui est l'entière responsabilité des femmes vouées dès lors à la plus stricte domesticité . Ce que Rousseau appelait «l'ombre» et «la clôture ${ }^{10} »-l^{\prime}$ intérieur, le privé, le sédentaire - est souhaité pour les femmes non pas tant à des fins morales (comme dans une perspective religieuse, par exemple) que dans une logique politique. Gardiennes du domaine privé, elles y accomplissent tout à la fois leur nature et leur destination sociale.

8 «Préface» de Femmes dans la Cité, 1815-1871, dir. Alain Corbin, Jacqueline Laouette, Michèle Rio-Sarcey, Grâne, Creaphis, 1997, p. 9.

9 Jean Bethke Elshtain a très bien expliqué que si les femmes sont responsables de la formation civilisatrice des enfants et parfois des hommes, «it follows, according to Rousseau's logic, that when women are unchaste, unfaithful, unseemly, vain, or frivolous, their ostensibly private behavior is suffused with public implications. Why? Because the basis of male public citizenship would disintegrate if his private world collapsed, as the citizen is also, necessarily, a husband-father, the head of a household", Public Man, Private Woman: Women in Social and Political Thought, Princeton, Princeton U.P., 1981, p. 161-62. On renvoie ici à l'ensemble des excellentes pages consacrées à Rousseau (p. 147-170) dans le chapitre IV.

10 Jean-Jacques Rousseau, Emile, ou de l'éducation, dans Oeuvres complètes, éd. B. Gagnebin et M. Raymond, Paris, Gallimard, «Bibliothèque de la Pléiade», 1969, vol. IV, p. 99. 
C'est donc pour la protection des jeunes filles et de la femme mariée que Mercier condamne non pas tant la prostitution en soi que le scandale public qu'elle constitue. Car le "publicisme» "se passe à la porte de l'honnête bourgeois qui a des filles, spectatrices de cet étrange désordre. Il leur est impossible de ne pas voir et de ne pas entendre ce que ces femmes licencieuses se permettent de dire» (CCCXXX, I, 876); «il est des tolérances publiques qu'il ne faut point du moins avouer publiquement; [...] Dès qu'il [le vice] sera voilé, il n'offensera plus l'ordre public» (CMLXIV, II, 1329) ${ }^{11}$. La femme qui s'offre en public au public érotise le regard, celui-là même qui se reporte sur toutes les figures de femmes décrites dans les rues, spectacles ou promenades de Paris. Cette érotisation autorise certains glissements entre femmes en général et prostituées en particulier, et une réflexion sur leur confusion possible: et si après tout il n'y avait pas de distinction naturelle possible? La généralisation dans le chapitre "Promenades publiques», "On s'aperçoit dans toutes ces promenades que les femmes ont grand besoin de voir et d'êtres vues» (CDXVIII, I, 1156), est précédée de considérations sur les filles entretenues, comme si l'évocation du sous-groupe «prostituées» amenait celle de la catégorie «femmes» en général. Au Palais-Royal, Lavater lui-même "aurait peine à distinguer une femme de condition, d'une fille entretenue» (CLXII, I, 381). Si les filles entretenues continuent de "se mettre très décemment», observe Mercier, «il faudra les connaître pour ne point se tromper, et pour les distinguer d'une honnête bourgeoise» (CDXVIII, I, 1156). L'amorale se moque de la morale, en en mimant l'attirail. La possibilité même de ces glissements réversibles équivaut à une condamnation oblique: l'érotisme flottant diffusé par toutes les femmes assoiffées de parures et de regards constitue un facteur de désordre en brouillant les signes identitaires sociaux et en produisant donc de constants détournements de sens et de désirs.

La bourgeoise (mère ou fille) seule peut «honnêtement» se produire en public à condition d'être rigoureusement entourée d'une sorte de garde personnelle, mari ou frères dirigeant ses pas à ses côtés: contrastant le Luxembourg au Palais-Royal, Mercier approuve cette "promenade sage, tranquille, solitaire, philosophique; là, l'honnête bourgeoisie offre ses filles pudiques, les mères y marchent décemment; les frères, sans en rougir, sont à côté de leur soeur; le quadragénaire donne le bras

11 C'est dans l'esprit de la position de Rétif de la Bretonne dans son ouvrage au titre-programme: Le Pornographe ou idées d'un honnête homme sur un projet de règlement pour les prostituées propre à prévenir les malheurs qu'occasionne le publicisme des femmes (1769). Voir l'hommage de Mercier à cet ouvrage: CMLXIV, II, 1329. 
à son épouse devenue enfin son amie», etc. (CMXIV, II, 1197-98). A ce modèle de la sortie familiale, où les femmes sont accompagnées de gardiens, est opposée la conduite de celles qui «sortent et vont seules dans les rues et sur les boulevards, la canne à la main» (XCIII, I, 236); «Les dames ont adopté, depuis peu, d'aller en cabriolet sans cavalier; elles y vont seules, elles y vont à deux, mais sans hommes; on dirait qu'elles voudraient changer de sexe» (DCCCLVI, II, 1043). Que deviennent ces éléments essentiels d'une féminité bien comprise, vulnérabilité et dépendance? Marchant ou roulant seules dans les rues, ces femmes "vulgivagues» ne ressemblent pas seulement aux prostituées ${ }^{12}$, mais aussi aux hommes, virilisation et prostitution étant communément liées dans la dénonciation de l'excessive liberté des femmes, comme le montre bien Geneviève Fraisse dans Muse de la raison à propos de Virey: «sortir de l'espace domestique revient à sortir de son sexe. Car la virilisation guette toute femme qui sort dehors pour quelque raison que ce soit, comme les femmes du peuple et les courtisanes ${ }^{13}$ ». Pas seulement: il est des femmes de la Cour qui «se sont fait homme: elles en ont pris l'habit pour vaguer à leur fantaisie; elles ont leurs courses, leurs affaires et leur causerie» auxquelles «les hommes sont devenus tout à fait étrangers» (DCCCLXIX, II, 1088). Que dire de ces "femmes-hommes", comme les nomme Mercier, qui «vont, viennent, sont partout» et «se mêlent de toutes les affaires» (DCCXLV, II, 708)? Le passage du spatial au moral puis au politique s'opère avec la plus grande facilité, sous l'autorité de Rousseau:

La remarque de Jean-Jacques Rousseau n'est que trop vraie, que les femmes à Paris, accoutumées à se répandre dans tous les lieux publics, à se mêler avec les hommes, ont leur fierté, leur audace, leur regard et presque leur démarche. Ajoutons que les femmes, depuis quelques années, jouent publiquement le rôle d'entremetteuses d'affaires. Elles écrivent vingt lettres par jour, renouvellent les sollicitations, assiègent les ministres, fatiguent les commis. Elles ont leurs bureaux, leurs registres; et à force d'agiter la roue de fortune, elles y placent leurs amants, leurs favoris, leurs maris, et enfin ceux qui les paient (CCXLIX, I, 624).

12 La femme se promenant seule, même et surtout l'aristocrate, semble appartenir de facto au vulgaire, selon le sens étymologique de vulgivague, de vulgus et vagari, errer. $\mathrm{AuXVIII}{ }^{\mathrm{e}}$ siècle le terme signifie prostituée, comme le montre Mercier lui-même: «On compte à Paris trente mille filles publiques c'est-à-dire, vulgivagues» (CCXXXVIII, I, 597).

13 Geneviève Fraisse, Muse de la raison: démocratie et exclusion des femmes en France, Paris, Gallimard, 1989; 1995, p. 155. 
Dans une condition moins élevée, dans les couches plus basses de la population, un autre problème: les célibataires, qui prolifèrent, se révèlent pleines de ressources et vivent infiniment mieux qu'elles ne le devraient. «La fille célibataire par choix, n'est point rare aujourd'hui dans l'ordre mitoyen. Des soeurs ou des amies s'arrangent pour vivre ensemble, et doubler leurs revenus en les plaçant en rentes viagères", ce qui leur permet de vivre «dans une espèce de liberté masculine», constate Mercier avec stupeur, «liberté qui, chez aucun peuple de la terre, ne fut le partage de leur sexe» (CCCIV, I, 796-797). Quant aux «filles du petit bourgeois, du simple artisan et du peuple [elles ont] toute liberté d'aller et venir» $\left(\right.$ CCXLVII, I, 619) ${ }^{14}$. Arlette Farge confirme: «[...] les façons de vivre à travers l'espace parisien [...] donnent à l'homme et à la femme de nombreuses occasions de se héler, se séduire ou se choisir. La fille du peuple travaille et circule autant que $l^{\prime}$ homme $[\ldots]^{15}$ ». Des femmes de condition aux «dames» et jusqu'aux filles du peuple, en passant par les célibataires de «l'ordre mitoyen», la contagion de la liberté de mouvement s'étend. Mais mises en mouvement dans la ville les femmes sont aussi mises en circulation, intégrées dans un cycle d'échanges. Sous cet angle, tout se passe comme si, seules en public dans l'espace urbain, elles signaient tacitement et obscurément un contrat qui les situerait d'emblée du côté de l'offre, offertes à ces demandeurs que seraient tous les passants.

"J'ai tant couru pour faire le Tableau de Paris, que je puis dire l'avoir fait avec mes jambes" (CMLVIII, II, 1309). Mercier est pleinement «peintre de la vie moderne» et rien de ce qui est urbain ne lui est étranger. $C^{\prime}$ est pourquoi le jugement moral porté de l'extérieur est le plus souvent suspendu, ou si mêlé et mitigé qu'il en perd ses contours rousseauistes. Les comportements et habitudes des femmes en public, leurs jeux de physionomie, leur démarche, leurs vêtements, leurs lieux de promenade et de travail, leurs moyens de transport, tout cela fascine Mercier. Cette fascination peut être d'ordre esthétique; la femme-spectacle, parée et offerte à la vue stimule un imaginaire érotisé où la pure jouissance du regard exclut toute condamnation. Le chapitre intitulé «Charmant coup d'oeil» par exemple est tout imprégné du plaisir de contempler les rangs des «jolies femmes» aux Champs-Elysées un beau jour de printemps: «la

14 Voir aussi DCXXVI, II, 338, sur l'autonomie des "grisettes».

15 A. Farge, La Vie fragile : violence, pouvoirs et solidarités à Paris au XVIII siècle, Paris, Hachette, 1986, p. 31. 
diversité des physionomies et des atours, la joie qu'elles ont d'être vues et de voir, [...] tout ajoute à ce tableau diversifié qui attache les regards, et fait naître mille idées sur ce que les modes enlèvent ou ajoutent à la beauté, sur l'art et la coquetterie des femmes, sur ce désir inné de plaire, qui fait leur bonheur et le nôtre» (LII , I, 142). Splendeur, donc, mais aussi misère. Mercier est souvent horrifié par le sort des femmes les plus pauvres, par les prostituées lamentables et désespérées ou ce qu'il appelle "le libertinage de détresse marchant dans les rues fangeuses» (CMLXIV, II, 1328). Extraordinairement sensible aux causes sociales et économiques de la prostitution, il repousse le carcan étroit et déformant du moralisme pour se livrer à de fréquentes analyses des rapports de force objectifs qui peuvent expliquer certains comportements, incluant cette prostitution. Il importe donc de souligner que l'austérité républicaine et la dureté morale qui peut l'accompagner ne dominent pas le Tableau. La passion de l'observation, la valorisation des circonstances spécifiques, l'attention au quotidien, le souci de justice et la reconnaissance de certains impératifs dans la vie des êtres qui l'entourent, tout cela nuance considérablement son point de vue sur la place des femmes dans les espaces publics de la vie urbaine.

Ce que Roche a nommé «la culture des apparences» ou la «sémiologie générale» dont parle Bonnet ${ }^{16}$ sont de première importance dans le Tableau. Les connexions à la fois réelles et symboliques entre les différents niveaux de la culture urbaine s'entrecroisent chez Mercier avec le discours moralement conservateur illustré plus haut pour former des configurations changeantes mais toujours visibles pour qui sait regarder. La composition fragmentaire du Tableau sert au mieux le jeu de recoupements et de découpages, de ruptures et de renouements établis entre différents paradigmes: sociaux, socio-psychologiques, économiques, culturels, politiques. Peut-être verra-t-on mieux cela à partir d'un objet exemplaire, la parure. Avant Balzac, avant Baudelaire, Mercier a étudié «les plus futiles détails de la mode et de l'habillement» «en eux-mêmes», et comme eux il en fait «des questions morales et philosophiques, car [ces détails] représentent la réalité immédiate dans son aspect le plus aigu, le plus agressif» et «le plus généralement vécu» comme l'écrit Caillois $^{17}$. Chantal Thomas a déjà et fort bien montré ce qui dans la mode

16 «La littérature et le réel», dans Jean-Claude Bonnet (édit.), Louis Sébastien Mercier: un hérétique en littérature, Paris, Mercure de France, 1995, p. 22. Voir aussi les pages 26-28 de cette même étude sur les «liaisons curieuses» que sait créer ou découvrir Mercier.

17 Le Mythe et l'homme, p. 163. 
a pu fasciner Mercier ${ }^{18}$. On se restreindra ici à la parure, elle-même fort complexe. Les linéaments suffiront. Première remarque: la prémisse est que son goût est une marque de la féminité en général: «Rien ne [...] touche plus vivement [la femme] que la parure» (CLXVIII, I, 395). Deuxième remarque: la parure a pour objet de susciter le désir en augmentant la beauté. Troisième remarque: la beauté est le capital des femmes; c'est elle qui mène les hommes à les épouser, à les entretenir ou au moins les payer pour qu'elles puissent assurer leur subsistance. La séduction (euphorique ou dysphorique) est un échange; dans le Tableau de Paris les femmes monnayent toujours, virtuellement au moins, leur beauté. Circulant dans la ville, s'exposant aux regards elles se constituent comme biens à désirer, et peut-être à acquérir. La parure pour les femmes mène au sexe qui mène à l'argent. On dira aussi qu'excepté l'honnête épouse bourgeoise mise hors circuit ou maintenue dans un circuit privé, toute femme parée en public est en spectacle et sur le marché de la séduction sexuelle, l'accent sur la vie extérieure et l'absence d'analyse qui porterait sur des individus particuliers éliminant dans le Tableau la dimension psychologique de la dite séduction.

Mais une fois établies ces propositions, on trouve en Mercier le patient dérouleur des multiples fils que l'écheveau "parure» contient en lui. La parure peut par exemple être l'instrument de la prostitution, ou son occasion, cause ou conséquence, symptôme ou incitation. Hors de toute condamnation morale, Mercier constate très crûment qu' «une pelisse se rend plus exigeante qu'un casaquin", ce qui explique pour les prostituées «la nécessité de se parer, afin d'être payées à un plus haut prix» (CCXXXVIII, I, 597-98). Par ailleurs, puisque «la dépense des modes excède aujourd'hui celle de la table et celle des équipages» (CLXXIII, I, 409), dans les maisons de proxénètes femmes «se rendent sourdement les petites bourgeoises et filles de boutique de toute espèce, qui, pour avoir des robes et soutenir leur parure, vont passer la soirée chez les matrones» (DXLII, II, 12). C'est que la compétition sexuelle entre les femmes est d'abord d'ordre social ${ }^{19}$ : «la petite bourgeoise veut imiter la

18 Chantal Thomas, «La sphère mouvante des modes», dans Louis Sébastien Mercier: un hérétique en littérature, 33-53.

19 Le thème n'est évidemment pas neuf. On lit dans les «Lettres sur les habitants de Paris» de Marivaux: "La vue d'une bourgeoise magnifique, quoique galante, va triompher de la vertu de cinquante de ses semblables qui la verront, et qui n'auront pas autant de parure qu'elle [...]. Ce qui gâte l'esprit des bourgeoises, c'est le faste continuel qui s'offre à leurs yeux: chaque équipage que rencontre en chemin une femme à pied porte en son cerveau une impression de douleur et de plaisir; de 
marquise et la duchesse. [...] et dans ce conflit de parures, la tête tourne réellement à nos femmes» (CLXXIII, I, 410-411). La critique de la frénésie de parure mène à des considérations sur le mariage. Dans la perspective du mari bourgeois que Mercier adopte occasionnellement, la femme assoiffée de parure ne s'y intéresse que parce qu'elle désire être vue, donc sortir de chez elle, donc abandonner son ménage. Le lien avec l'argent est ici inverse de ce qu'il peut être lorsque la courtisane parée est paradée par son amant dans un étalage ostentatoire de sa richesse. Le mari bourgeois est au contraire poussé à dépenser pour quelque chose dont il ne veut pas: les sorties publiques de sa femme: "Le pauvre mari est obligé de suer sang et eau pour satisfaire aux caprices de son épouse. Elle ne revient point $\mathrm{d}^{\prime}$ une promenade sans avoir une fantaisie nouvelle» (CLXXIII, I, 410). Les prédicateurs aussi tonnent contre la dissipation dévoilée par le goût de la parure. Mais parure, exhibition publique, érotisme et argent font de nouveau une apparition liée, apparition surprise lorsque Mercier fait le portrait d'une jolie quêteuse, engagée par un «sévère pasteur» pour augmenter les revenus de l'église. «Il a prêché le matin contre la parure, il a appelé scandale effroyable tous les ornements légers qui ajoutent à la beauté. Le soir, il attend d'une aimable quêteuse qu'il a invitée, de sa taille élégante et de son joli minois, la récolte d'aumônes plus abondantes. Elle est parée; son sein est découvert», l'éloquence de son bras nu est irrésistible, etc. (CXXXIII, I, 318).

Si l'on se tourne du côté du monde du travail, par contre, la parure comme un des domaines de l'industrie du luxe est valorisée parce qu'elle offre de nombreuses possibilités d'emplois. Mercier peut lier de nouveau parure, argent et érotisme en proposant des réflexions (nombreuses dans le Tableau) sur les marchandes de mode et les "grisettes ${ }^{20}$ ». "On appelle grisette la jeune fille qui, n'ayant ni naissance ni bien, est obligée de travailler pour vivre, et n'a d'autre soutien que l'ouvrage de ses mains. Ce sont les monteuses de bonnets, les couturières, les ouvrières en linge, etc., qui forment la partie la plus nombreuse de cette classe» (DCXXVI,

douleur, en se voyant à pied; de plaisir, en se figurant celui qu'elle aurait si elle possédait une pareille voiture. Le moyen que le cerveau d'une femme tienne à cela?», dans Journaux et oeuvres diverses, éd. F. Deloffre et M. Gilot, Paris, Bordas, coll. Garnier, 1988, p. 20.

20 Sur le milieu des marchandes de mode et des grisettes, voir Jennifer Jones, "Coquettes and Grisettes: Women Buying and Selling in Ancien Régime France», dans Victoria de Grazia et Ellen Furlough (édit.), The Sex of Things: Gender and Consumption in Historical Perspective, Berkeley/Los Angeles, University of California Press, 1996, 25-53. Jones cite d'ailleurs assez fréquemment le Tableau de Paris. 
II, 337). Or voici comment débute un chapitre intitulé «Est-ce un sérail?»: «L'idée d'un sérail prend à tout étranger qui voit pour la première fois une boutique meublée de marchandes de modes; il y a des minois charmants à côté de laides figures assises dans un comptoir à la file l'une de l'autre; elles ornent ces pompons, ces colifichets que la mode varie; on les lorgne en passant. Ces filles, l'aiguille à la main, jettent incessamment l'oeil dans la rue. [...] [Elles] se réjouissent à considérer les passants, et s'imaginent voir autant d'amants» (DCCCLXXXI, II, 1113). Circulation des biens, circulation des personnes: Mercier observe les moeurs de celles qui sont "obligées de travailler pour vivre», les comprend et laisse Rousseau loin derrière lui.

Le décalage est assez substantiel entre le discours bourgeois et masculin normatif sur les femmes tel que Geneviève Fraisse par exemple l'a analysé chez les contemporains pré- et post-révolutionnaires de Mercier (comme Roussel, Maréchal ou Cabanis), discours qui est déjà nettement présent dans le Tableau, et le considérable ensemble descriptif, incluant tant de situations, tant de cas que ce discours ne peut absorber sans les trahir. Au bout du compte, ce sont surtout les riches aristocrates paradant amants et diamants, les courtisanes entretenues à grands frais ou encore les prostituées agressives que condamne Mercier. Dans l'immense espace médian, il déploie les gradations, trace de fines lignes d'un état à l'autre, voire d'un hasard à un autre, de sorte que l'essence «femme» s'y volatilise. C'est qu'il dévoile involontairement un aspect idéologique de l'analyse rousseauiste qui sous le nom de «femme» $\mathrm{n}$ 'inclut en fait que les femmes de condition bourgeoise. Dans l'esprit de l'épigraphe du tome IX, Mercier fait descendre du ciel la philosophie et l'installe dans les villes ${ }^{21}$. Faisant place aux femmes du peuple, il élargit nécessairement, complique et surtout concrétise la perspective sur la présence des femmes dans les espaces publics de la ville, et dans les rues en particulier. Comment prescrirait-on à ces centaines de milliers de Parisiennes un rôle et une fonction uniques et universels? Après tout, comme l'écrit bizarrement (ou naïvement) Mercier, «Songez donc que les vierges et les honnêtes femmes passent aussi dans les rues» (CDLXXXV, I, 1324). A quoi donc songeait Rousseau?

$$
* * *
$$

21 L'épigraphe en latin au frontispice du tome IX est tirée des Tusculanes de Cicéron (liv. V, IV, 10) et est ainsi traduite dans l'édition Bonnet: «Socrate le premier invita la philosophie à descendre du ciel, l'installa dans les villes, l'introduisit jusque dans les foyers, et lui imposa l'étude de la vie, des moeurs, des choses bonnes et mauvaises» (II, 1685). 
C'est une vision en kaléidoscope qu'offre Mercier. Sa valeur n'est pas dans sa cohérence ni dans la profondeur de son champ mais dans ses alternances rapides, sa mobilité, ses combinaisons infiniment renouvelées, ses pouvoirs d'accueil aussi. L'énormité du livre et ses constantes brisures en brefs chapitres ouverts sur tous les sujets possibles et imaginables interdisent qu'y domine un ordre cohérent et systématique, autant structurel que moral. Certes, défenseur d'un ordre moral bourgeois, on ne saurait nier que Mercier l'est lorsqu'il exprime le voeu que les femmes restent à la maison car telle est leur nature ${ }^{22}$. Sous cet angle toute femme qui sort sans un lourd encadrement familial est suspecte. Mais Mercier est aussi l'analyste dégagé et fin des pulsions et impulsions de la culture urbaine dont les femmes sont partie intégrante. Le désir stimulé par l'érotisation de la vie en public engendrée par leur présence produit un autre type d'ordre, moteur irrépressible des échanges culturels et d'une production économique intensive, dans l'industrie du luxe en particulier. D'autre part, l'inclusion du peuple oblige Mercier à nuancer, voire à oblitérer certaines de ses positions morales: la femme du peuple au travail a de toute évidence sa place nécessaire et légitime dans la rue, dans les boutiques, aux côtés des hommes. C'est pourquoi le Mercier moraliste, les yeux troublés par le spectacle omniprésent de la prostitution, peut bien affirmer souhaiter l'invisibilité des femmes dans Paris; le sociologue intelligent qu'il est aussi, et surtout l'écrivain, leur donne pleinement droit de cité.

\section{LAURENCE MALL}

University of Illinois at Urbana-Champaign

22 Si les femmes «ne sont jamais bien dans toutes ces courses», c'est "qu'elles veulent être absolument où la nature ne veut pas qu'elles soient» (LIV, I, 146), c'est-à-dire hors de leur maison. Sur le Mercier bourgeois traditionnel à l'égard des femmes, voir l'article ancien mais toujours intéressant de Christiane Berkowe, «Louis-Sébastien Mercier et les femmes," The Romanic Review, 55, 1, February 1964, 17-29. 